\title{
Менопаузальная гормональная терапия: новый взгляд на старые проблемы
}

\author{
Ю.С. Драпкина, ORCID: 0000-0002-0545-1607, e-mail: julia.drapkina@gmail.com
}

Национальный медицинский исследовательский центр акушерства, гинекологии и перинатологии имени академика В.И. Кулакова; 117997, Россия, Москва, ул. Академика Опарина, д. 4

\section{Резюме}

Наиболее эффективный способ коррекции симптомов климактерия заключается в назначении менопаузальной гормональной терапии (МГТ). Было показано, что своевременно начатая МГТ позволяет не только уменьшить частоту и интенсивность вазомоторных нарушений, но и существенно снизить риск остеопороза и ишемической болезни сердца (ИБС). Однако профиль безопасности МГТ был поставлен под сомнение после полученных результатов о повышении риска венозной тромбоэмболии, инсульта и рака молочной железы на фоне приема МГТ. Несмотря на то что полезные свойства МГТ значительно превышают возможные риски развития осложнений, современные международные руководства рекомендуют использовать минимальные дозы МГТ. Низкодозированные и ультранизкодозированные режимы МГТ позволяют сохранить эффективность в отношении симптомов менопаузы, но при этом снизить частоту возникновения нежелательных побочных реакций до минимума.

Ключевые слова: климактерий, менопаузальная гормональная терапия, дидрогестерон, эстрадиол

Для цитирования: Драпкина Ю.С. Менопаузальная гормональная терапия: новый взгляд на старые проблемы. Медицинский совет. 2019;(13):138-141. doi: 10.21518/2079-701Х-2019-13-138-141.

Конфликт интересов: автор заявляет об отсутствии конфликта интересов.

\section{Menopausal hormone therapy: new insights into the old problems}

Yulia S. Drapkina, ORCID: 0000-0002-0545-1607, e-mail: julia.drapkina@gmail.com

Federal State Budgetary Institution «National Medical Research Center of Obstetrics, Gynecology and Perinatology named after Academician V.I. Kulakov» of the Ministry of Health of the Russian Federation: 117997, Russia, Moscow, 4, Oparina str.

\begin{abstract}
The most effective symptomatic treatment of menopause is menopausal hormone therapy (MHT). It has been shown that MHT administered in proper time can not only reduce the frequency and intensity of vasomotor disorders, but also significantly reduce the risk of osteoporosis and coronary heart disease (CHD). However, several research showed the increased risk of venous thromboembolism, stroke and breast cancer in patients receiving MHT, thereby MHT safety was questioned. Despite the fact that the beneficial properties of MHT significantly exceed the possible risks of side effects, modern international guidelines recommend to use minimal doses of MHT. Low-dose and ultra-low dose MHT regimens make it possible to maintain effectiveness, but at the same time reduce the incidence of undesirable side reactions to a minimum.
\end{abstract}

Keywords: menopause, menopausal hormone therapy, didrogesterone, estradiol

For citing: Drapkina Yu.S. Menopausal hormone therapy: new insights into the old problems. Meditsinskiy sovet = Medical Council. 2019;(13):138-141. (In Russ.) doi: 10.21518/2079-701X-2019-13-138-141.

Conflict of interest: The author declare no conflict of interest.

\section{ВВЕДЕНИЕ}

Репродуктивный потенциал женщины регулируется эстрогенами и прогестероном - гормонами, образующимися в яичниках. При старении женской репродуктивной системы происходит постепенное снижение гормонпродуцирующей функции яичников, что приводит к дефициту половых гормонов. В 2001 г. была впервые предложена клинико-гормональная характеристика этапов старения репродуктивной системы уженщины (Stages of Reproductive Aging Workshop - STRAW). Данная классификация была пересмотрена в 2011 г. с учетом новых результатов крупных исследований (STRAW+10). Согласно классификации STRAW+10, выделяют 4 периода климактерия [1]:

Период менопаузального перехода (характеризуется вариабельностью циклов, начинается в 40-45 лет и заканчивается менопаузой)

Менопауза (полное отсутствие менструаций более 12 месяцев)

- Перименопауза (период менопаузального перехода и 12 месяцев после последней менструации)

- Постменопауза (период после менопаузы). 
Возрастная перестройка организма характеризуется многочисленными симптомами разной степени выраженности, однако среди наиболее частых проявлений следует отметить [2]:

- Нерегулярный менструальный цикл

Потливость и приливы

Сердцебиение

Сухость во влагалище

Раздражительность, депрессия, дисфория

Диспареуния

- Недержание мочи, цисталгии

Бессонница.

Эстрогенный дефицит может не только снижать качество жизни пациентки, но и приводить к серьезным осложнениям. На фоне снижения эстрогенов повышается риск развития остеопороза, болезни Альцгеймера, ИБС [3]. Таким образом, менопауза диктует необходимость коррекции гипоэстрогении с помощью МГТ.

Основные показания к назначению МГТ включают коррекцию вазомоторных нарушений, урогенитальных расстройств и профилактику остеопороза. Известно много противоречивых данных в отношении безопасности МГТ. Результаты исследования Women's Health Initiative (WHI) показали, что назначение МГТ повышает риск рака молочной железы, инсульта и венозной тромбоэмболии [4].

Стоит отметить, что задача МГТ состоит не в восстановлении физиологической концентрации эстрадиола, характерной для молодых женщин, а в терапии симптомов климактерия. Именно поэтому при выборе оптимального режима МГТ стоит отдавать предпочтение низким дозам эстрогенов, которые будут иметь минимальный риск побочных эффектов. Кроме этого, сочетание низких доз эстрогенов с природным прогестероном или дидрогестероном позволяет защитить эндометрий от гиперпластических процессов или канцерогенеза при интактной матке [5]. В отличие от других аналогов прогестерона, дидрогестерон не обладает эстрогенной, андрогенной, анаболической или глюкокортикостероидной активностью [6].

Результаты многих исследований (Stevenson J.C. et al, 2001; Panay N., 2007) показали, что назначение 17ß-эстрадиола и дидрогестерона заметно уменьшало выраженность приливов, потливости, недержания мочи, диспареунии, а также эффективно предотвращало развитие остеопороза. Для того чтобы снизить риски развития осложнений и получить максимальные преимущества от МГТ необходим персонифицированный подход к выбору оптимального режима лечения [7]. На сегодняшний день широко распространены 4 комбинации МГТ в зависимости от дозы эстрогенового и прогестеронового компонента.Для фазы менопаузального перехода рекомендуется использовать 1 или 2 мг 17ß-эстрадиола в зависимости от выраженности симптомов и специфических факторов риска в комбинации с 10 мг дидрогестерона в циклическом режиме. Для пациенток в постменопаузе предпочтительнее назначение ультранизкодозированной пероральной терапии, включающей 0,5 мг 17ß-эстрадиола в сочетании с 2,5 мг дидрогестерона или 1 мг 17 -эстрадиола с 5 мг дидрогестерона в зависимости от клинической ситуации.

\section{ВЛИЯНИЕ МГТ НА ВАЗОМОТОРНЫЕ НАРУШЕНИЯ}

Было проведено двойное слепое многоцентровое рандомизированное исследование, в которое вошли 313 женщин: 124 пациентки принимали 17 $\beta$-эстрадиол в дозе 0,5 мг и дидрогестерон в дозе 2,5 мг (группа I); 62 женщины, получающие 17 $\beta$-эстрадиол - 1 мг и дидрогестерон - 5 мг, были распределены в группу II и 127 пациенток - в контрольную группу III, получающую плацебо [8]. Продолжительность исследования составляла 13 недель. Спустя 13 недель группа III перешла на МГТ, включающую 0,5 мг эстрадиола и 2,5 мг дидрогестерона в течение 39 недель. Остальные пациенты принимали лечение без изменения дозы. Через 13 недель терапии было обнаружено значительное снижение выраженности приливов в группе । (ультранизкодозированная терапия) по сравнению с группой III (плацебо) (-6,4 vs -4,9, p<0,001). Эффективность ультранизкодозированной терапии была сопоставима с группой II (17 $\beta$-эстрадиола - 1 мг + дидрогестерона - 5 мг). Все участницы исследования заполняли Шкалу симптомов менопаузы (ШСМ) для оценки качества жизни, обусловленного состоянием здоровья. В I и II группе также отмечалось значительное улучшение показателей качества жизни.

Результаты еще одного исследования показали, что частота приливов также влияет на когнитивную функцию. Чем чаще отмечались приливы, тем более выраженными были нарушения памяти и другие когнитивные расстройства [9]. Таким образом, снижение выраженности и частоты приливов с помощью МГТ позволяет в дополнение предупреждать когнитивные нарушения.

\section{ВЛИЯНИЕ МГТ НА РИСК РАЗВИТИЯ ОСТЕОПОРОЗА}

Биосинтез кальцитонина, синтезируемый парафолликулярными клетками щитовидной железы, зависит от уровня эстрогенов. Кальцитонин тормозит резорбцию костной ткани и высвобождение из нее кальция и фосфата. В состояние гипоэстрогении дефицит данного гормона приводит к быстро прогрессирующему остеопорозу. Именно поэтому эстрогенный компонент в составе МГТ предотвращает потерю костной массы, связанную с менопаузой [10]. Своевременно назначенная МГТ может снизить риск переломов практически на $30 \%$. Международное общество по менопаузе (International Menopause Society) рекомендует применение МГТ в качестве препарата первой линии для профилактики остеопороза у пациенток в постменопаузе моложе 60 лет.

Результаты многоцентрового двойного слепого проспективного рандомизированного плацебо-контролируемого исследования (Lees B. Et al, 2001) показывают значительные преимущества назначения 1 или 2 мг 17 $\beta$-эстрадиола в сочетание с дидрогестероном, по сравнению с плацебо, в профилактике переломов поясничного отдела позвоночника, шейки бедренной кости и снижения плотности костной ткани по данным денситометрии. Обе дозы эстрадиола эффективно сохраняют минеральную плотность костной ткани, при этом процент положительного ответа на лечение более низкой дозировкой у женщин старше 59 лет остается 
таким же, как и при применении более высокой дозировки у женщин моложе 59 лет. Таким образом, ультранизкодозированная МГТ может быть рекомендована пациенткам более старшего возраста для минимизации побочных эффектов и оптимизации специфических преимуществ.

Существуют противоречивые данные, что дидрогестерон может снижать положительное действие эстрадиола на костную ткань. В исследовании приняло участие 26 женщин в постменопаузе, получавшие ежедневно 2 мг эстрадиола или 2 мг эстрадиола в комбинации с дидрогестероном. Было обнаружено, что в группе, принимавшей только эстрадиол, отношение остеокальцин/дезоксипиридинолин и отношение С-терминальный пропептид проколлагена І/дезоксипиридинолин было значительно выше. Ученые предположили, что дидрогестерон снижает положительный эффект эстрогенового компонента на минеральную плотность костей. Однако более свежие результаты недавних исследований не смогли подтвердить отрицательное влияние дидрогестерона на ремоделирование костной ткани [11].

\section{ВЛИЯНИЕ МГТ НА СЕРДЕЧНО-СОСУДИСТЫЕ ЗАБОЛЕВАНИЯ}

Сердечно-сосудистые заболевания к 60 годам встречаются у 80\% женщин. Такое широкое распространение данной группы заболеваний у женщин в постменопаузе обусловлено исчезновением защитного влияния эстрогенов на функцию эндотелия, кардиомиоциты, гладкомышечные клетки сосудов. Исследование WHI (The Women's Health Initiative Steering Committee, 2004) показало повышение риска возникновения тромбоэмболии и инсульта на фоне МГТ, тем самым поставив под сомнение безопасность терапии эстрогенами и дидрогестероном в отношенении сердечно-сосудистой системы. Однако при повторном анализе результатов WHI было обнаружено, что спустя 13 лет приема МГТ женщинами от 50 до 59 лет происходит снижение риска инфаркта миокарда и общей смертности от сердечно-сосудистых причин. Кроме этого, на фоне приема МГТ пациенткам с прегипертензией и гипертензией реже требовалась антигипертензивная терапия (Кауа C et al., 2007).

При раннем назначении МГТ (в первые 6-8 лет после наступления менопаузы) снижалась смертность и частота госпитализации по поводу инфаркта миокарда и сердечной недостаточности, и подобный эффект сохранялся в течение 6 лет после отмены МГТ. Таким образом, для адекватной профилактики сердечно-сосудистых заболеваний необходимо придерживаться правила «терапевтического окна». Применение МГТ для снижения смертности и предупреждения инфаркта миокарда наиболее эффективно у пациенток до 60 лет, с продолжительностью менопаузы не более 10 лет [12].

\section{ВЛИЯНИЕ МГТ НА РИСК ВЕНОЗНОЙ ТРОМБОЭМБОЛИИ}

Известно, что пероральный режим МГТ увеличивает риск венозной тромбоэмболии. Однако исследование, в котором приняло участие 1006 женщин в возрасте от 45-58 лет с недавно наступившей менопаузой, показало любопытные результаты. В контрольной группе 504 женщины получали плацебо, а в исследуемой группе 502 пациентки принимали МГТ в течение 11 лет. Период наблюдения за участницами исследования составил 16 лет. Было обнаружено, что у пациенток, начавших принимать МГТ сразу после начала менопаузы и продолжающих получать это лечение в течение 10 лет, уменьшалась смертность от сердечно-сосудистых заболеваний или инфаркта миокарда, а риск развития венозной тромбоэмболии или инсульта не увеличивался [12]. Эти данные позволяют по-новому взглянуть на риск венозной тромбоэмболии у пациенток, получающих МГТ.

Стоит отметить, что состояние гипоэстрогении в менопаузе также ассоциировано со снижением чувствительности к инсулину и отмечается понижением толерантности к глюкозе. Было показано, что своевременное назначение перорального эстрадиола в составе МГТ снижает частоту развития сахарного диабета 2-го типа на $35 \%$ у женщин в постменопаузе, страдающих сердечно-сосудистыми заболеваниями (Kanaya A.M. et al., 2003).

\section{ВЛИЯНИЕ МГТ НА МОЛОЧНЫЕ ЖЕЛЕЗЫ}

Результаты исследования WHI показало увеличение риска рака молочной железы при использовании комбинированной МГТ (Chlebowski R.T. et al., 2003). При более детальном рассмотрение было обнаружено, что у женщин, которые 5 лет получали только эстрогены, наблюдалось существенное снижение риска развития рака молочной железы [13]. Полученные результаты позволили предположить, что на риск развития злокачественных новообразований молочной железы влияет прогестероновый компонент в составе МГТ.

Существуют убедительные данные, что различные прогестагены, входящие в состав МГТ, по-разному влияют не только на риск развития рака молочной железы, но и на такие побочные эффекты, как болезненность молочных желез. Было показано, что в отношении масталгии наиболее благоприятным профилем обладает сочетание эстрадиола с дидрогестероном [14].

Профиль риска таких прогестагенов, как прогестерон и дидрогестерон, может отличаться от профиля риска «синтетических» прогестинов. В крупном когортном французском исследовании было показано, что риск инвазивного рака молочной железы повышается при длительном применении комбинаций синтетического прогестагена [15]. Однако для дидрогестерона, входящего в состав МГТ, такого побочного эффекта обнаружено не было ( $p=0,16)$, несмотря на то, что эффекты дидрогестерона и прогестерона очень похожи. Другие экспертные оценки по Базе данных семейных врачей Великобритании (UK General Practitioners Research Data Base - GPRD) также не обнаружили повышения риска рака молочной железы при приеме МГТ, включающей эстрадиол и дидрогестерон [16]. Результаты еще одного исследования in vitro (Franke H.R., Vermes I., 2003) подтверждают, что такие прогестагены, как медроксипрогестерона ацетат, норэтистерона ацетат и диеногест в комбинации с эстрадиолом или вне комбинации оказывают стимулирующее влияние на пролиферацию клеток и 
повышают риск рака молочной железы, в то время как дидрогестерон, тиболон или прогестерон не обладают таким действием. Учитывая полученные данные, можно предположить, что ультра- и низкодозированная пероральная МГТ, включающая эстрадиол в комбинации с дидрогестероном, обладает наилучшим профилем безопасности в отношении развития рака молочных желез и масталгии.

\section{ВЛИЯНИЕ МГТ НА ЭНДОМЕТРИЙ}

Ультра- и низкодозированная МГТ в непрерывном режиме связана с лучшим профилем кровотечений по сравнению со стандартными дозами эстрадиола и дидрогестерона [8].

Было показано, что профиль кровотечений был лучше в группе пациенток, получающих ультранизкодозированную МГТ (17ß-эстрадиол 0,5 мг + дидрогестерон 2,5 мг) с меньшим числом дней с кровотечениями/мажущими выделениями, чем в группе пациентов, принимающих эстрадиол в дозе 1 мг + дидрогестерон 5 мг. При этом общий процент отсутствия менструаций при приеме ультранизкодозированной МГТ составлял $81 \%$ и увеличивался до 91\% спустя 1 год приема [8].

Результаты еще одного исследования подтверждают, что ультранизкодозированная МГТ обладает надежным профилем безопасности в отношении риска развития гиперплазии и рака эндометрия [17]. Безопасность данного вида лечения подтверждена Руководством Комитета по контролю за оборотом медицинской продукции, предназначенной для людей (Committee for Medicinal Products for Human Use, CHMP).

\section{ЗАКЛЮЧЕНИЕ}

Таким образом, профиль безопасности МГТ зависит от состава препарата и дозы, входящих в него компонентов. Ультранизкодозированная и низкодозированная МГТ, включающая комбинацию $17 \beta$-эстрадиола и дидрогестерона, эффективно снижает вазомоторные симптомы и положительно влияет на качество жизни. Кроме этого, МГТ позволяет предупредить развитие тяжелых сердечно-сосудистых заболеваний, когнитивных расстройств, а также приводит к снижению риска остеопороза и переломов. Профиль безопасности комбинации 17 $\beta$-эстрадиола и дидрогестерона соответствует современным требованиям.

Следует подчеркнуть, что для получения максимального профилактического и терапевтического эффекта МГТ должна быть назначена в «окно терапевтического воздействия», которое включает фазу менопаузального перехода и/или первые 3-5 лет постменопаузы. Более позднее назначение МГТ неэффективно и сопряжено с более высоким риском развития осложнений. Рекомендуется применять МГТ в течение 5-7 лет под динамическим контролем врача. Появление в арсенале у врачей ультранизкодозированной и низкодозированной пероральной МГТ может существенно увеличить приверженность пациенток к данному режиму лечения и повысить продолжительность жизни.

Поступила / Received 26.06.2019 Отрецензирована / Review15.07.2019 Принята в печать / Accepted 05.09.2019

Список литературы / References

1. Harlow S.D., Gass M., Hall J.E., et al. Executive summary of the Stages of Reproductive Aging Workshop +10 : addressing the unfinished agenda of staging reproductive aging. Climacteric. 2012;15(2):105-114

2. Hoffman B. Williams Gynecology. New York: McGraw-Hill Medical. 2012:555-56.

3. ESHRE Capri Workshop Group. Perimenopausal risk factors and future health. Human Reproduction Update. 2011;17(5):706-17.

4. Boardman H.M., Hartley L., Eisinga A., Main C., Roqué i Figuls M., Bonfill Cosp X., Gabriel Sanchez R, Knight B. Hormone therapy for preventing cardiovascular disease in post-menopausal women. The Cochrane Database of Systematic Reviews. 2015 Mar 10(3).

5. North American Menopause Society. Estrogen and progestogen use in postmenopausal women: 2010 position statement of The North American Menopause Society. Menopause. 2010;(17):242-255.

6. Regidor P.A. Progesterone in Peri- and Postmenopause: A Review. Geburtshilfe Frauenheilkd. 2014;74(11):995-1002.

7. Сметник А.A. Менопаузальная гормонотерапия с дидрогестероном: аспекты эффективности и безопасности ультранизких доз. Медицинский совет. 2017;(2):92-99.
Smetnik A.A. Menopausal hormone therapy with dydrogesterone: aspects of efficacy and safety of ultra-low doses. . Medicinskij sovet = Medical Council. 2017;(2):92-99. (In Russ.) doi: 10.21518/2079-701X-2017-2-92-99.

8. Stevenson J.C., Durand G., Kahler E. et al. Oral ultralow dose continuous combined hormone replacement therapy with $0.5 \mathrm{mg}$ 17beta- oestradiol and 2.5 mgdydrogesterone for the treatment of vasomotor symptoms: results from a double-blind, controlled study. Maturitas, 2010;(67):227-32.

9. Maki P.M., Drogos L.L., Rubin L.H., Banuvar S., Shulman L.P., Geller S.E. Objective hot flashes are negatively related to verbal memory performance in midlife women. Menopause. 2008 Sep-Oct;15(5):848-56.

10. Gambacciani M., Cappagli B., Ciaponi M., Pepe A., Vacca F., Genazzani A.R. Ultra low-dose hormone replacement therapy and bone protection in postmenopausal women. Maturitas. 2008;(59):2-6.

11. Stevenson J.C., Panay N., Pexman-Fieth C. Oral estradiol and dydrogesterone combination therapy in postmenopausal women: Review of efficacy and safety. Maturitas. 2013;(760:10-21.

12. Schierbeck L.L., Rejnmark L., Tofteng C.L. et al. Effect of hormone replacement therapy on cardiovascular events in recently postmenopausal women: randomised trial. British Medical Journal. 2012;(345):e6409.
13. Anderson G.L., Chlebowski R.T., Aragaki A.K., et al. Conjugated equine oestrogen and breast cancer incidence and mortality in postmenopausal women with hysterectomy: extended follow- up of the Women's Health Initiative randomisedplacebo-controlled trial. The Lancet Oncology. 2012:(13):476-86.

14. Khalid Rida Murshid. A Review of Mastalgia in Patients with Fibrocystic Breast Changes and the Non-Surgical Treatment Options. Journal of Taibah University Medical Sciences. 2011;6(Issue 1):1-18.

15. Fournier A., Berrino F., Clavel-Chapelon F. Unequal risks for breast cancer associated with different hormone replacement therapies: results from the E3N cohort study. Breast Cancer Res Treat. 2008;(107):103-111.

16. Schneider C., Jick S.S., Meier C.R. Risk of gynecological cancers in users of estradiol/dydrogesterone or other HRT preparations. Climacetric. 2009:1-11 ([Epub ahead of print]).

17. Bergeron C., Nogales F.F., Rechberger T. et al. Ultra low dose continuous combined hormone replacement therapy with $0.5 \mathrm{mg} 17$ beta-oestradiol and 2,5 mg dydrogesterone: protection of the endometrium and amenorrhoea rate. Maturitas. 2010;66(2):201-205.

\section{Информация об авторе:}

Драпкина Юлия Сергеевна, врач акушер-гинеколог, аспирант, Федеральное государственное бюджетное учреждение «Национальный медицинский исследовательский центр акушерства, гинекологии и перинатологии имени академика В.И. Кулакова» Министерства здравоохранения Российской Федерации; тел.: +7(916) 950-07-45; e-mail: julia.drapkina@gmail.com

\section{Information about the author:}

Drapkina Yulia Sergeevna, Obstetrician-Gynecologist, Postgraduate Student, Federal State Budgetary Institution «Kulakov National Medical Research Centre for Obstetrics, Gynaecology and Perinatology» of the Ministry of Health of the Russian Federation; Tel.: +7(916) 950-07-45; e-mail: julia.drapkina@gmail.com 